
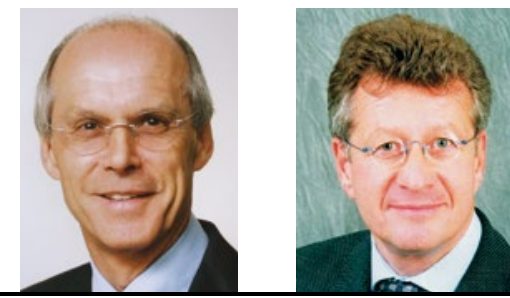

Dr. med. P. Stiefelhagen DRK-Klinikum Westerwald, Hachenburg

\title{
Hirnarterienaneurysma
}

\section{Wie lange hält das Clipping im Gehirn?}

? Dr. Sigrid Schubert, München: Ich habe einen 52-jährigen Patienten, bei dem 1998 nach einem rupturierten Aneurysma der A. communicans anterior ein Clipping durchgeführt wurde. Die Folgen der Hirnblutung sind vollständig abgeklungen. Wie lange hält so ein Clipping? Muss irgendwann nachoperiert werden? Und wie hoch ist 18 Jahre nach Setzen des Clippings das Risiko einer Blutung im Bereich des Clippings bei schwerer körperlicher Betätigung oder Sex?

Der Patient ist abgesehen von einer primären Hypercholesterinämie gesund und voll arbeitsfähig. Sein Cholesterinwert liegt trotz einer maximalen Simvastatin-Dosis - der Patient hat bereits passagere Muskelschmerzen - bei > $450 \mathrm{mg} / \mathrm{dl}$.

! MW-Experte Stiefelhagen: Diese Clips müssten lebenslang halten. Bisher gibt es auch keine Berichte über etwaige Rezidivblutungen.

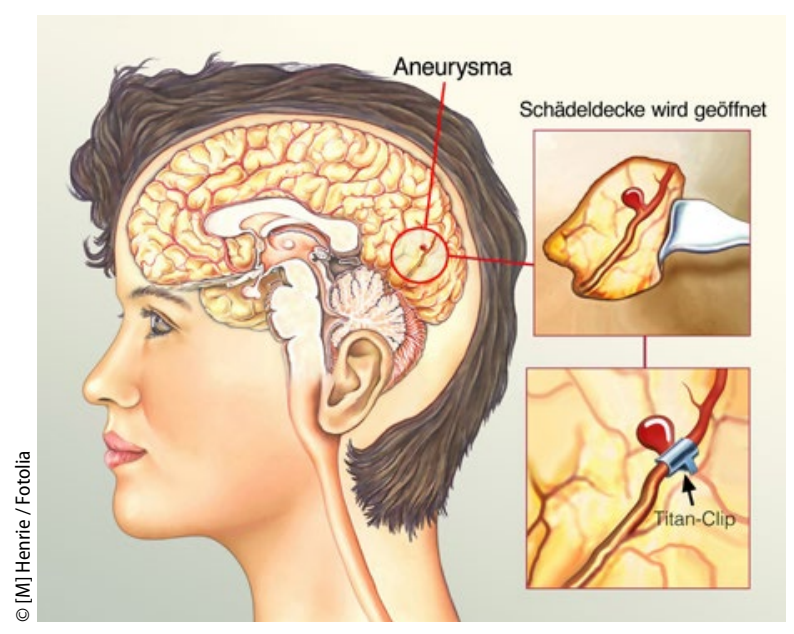

Der Clip auf den Hals des Aneurysmas unterbindet den Blutzustrom in die Aussackung vollständig.

\begin{abstract}
MMW-Experte FüeßI:
Der Clip ist nicht das Problem, er hält ein Leben lang. Allerdings haben $\mathrm{Pa}$ tienten mit einem Aneurysma ein erhöhtes Risiko, ein weiteres zu bekommen. Mit diesem Wissen zu leben, kann ein Problem darstellen. Sich deshalb nie mehr körperlich anzustrengen oder keinen Sex mehr zu haben, ist auch nicht praktikabel. Für die Lebensqualität erscheint mir Verdrängung noch am besten zu sein.
\end{abstract}

\section{Hirnaneurysma: Score zeigt Dringlichkeit der Operation an}

Aus Studien sind viele Risikofaktoren bekannt, die eine Ruptur intrakranieller Aneurysmen begünstigen - etwa das weibliche Geschlecht, Tabakabhängigkeit und jüngeres Alter. Im letzten Jahr hat PD Dr. Nima Etminan von der Universität Düsseldorf mit 68 Spezialisten aus aller Welt einen Praxisscore entwickelt, der Ärzten rasch eine Therapieempfehlung liefert. Der Fragebogen für den „Unruptured Intracra- nial Aneurysm Treatment Score" (UIATS) ist umfangreich, lässt sich aber noch auf einer DIN-A4-Seite abbilden.

Einige der Risikofaktoren liefern Punkte für einen invasiven Eingriff. Dazu zählen alle Aneurysma-Charakteristika sowie Patientenangaben wie Alter, klinische Symptome und Lifestyle. Die anderen Faktoren summieren sich zu Punkten für ein konservatives Vorgehen. Dazu gehören sämtliche therapie-, aber auch einige patientenbezogenen Parameter wie Komorbiditäten und Lebenserwartung. Es ergeben sich zwei unterschiedliche Werte. Liegt der Unterschied bei drei oder mehr Punkten, ergibt sich eine Therapieempfehlung. Die Evaluation muss bei multiplen Aneurysmen für jede Aussackung einzeln erfolgen. _ _ mut

- Etminan Net al. Neurology. 2015;85:881-9 